\title{
Neue Definitionen der Integrativen Medizin: Alter Wein in neuen Schläuchen?
}

\author{
Tobias Esch $^{\mathrm{a}}$ Benno Brinkhaus ${ }^{\mathrm{b}}$ \\ a Institut für Integrative Gesundheitsversorgung und Gesundheitsförderung, Universität Witten/Herdecke, \\ Witten, Deutschland; ${ }^{\mathrm{b}}$ Komplementäre und Integrative Medizin, Institut für Sozialmedizin, Epidemiologie und \\ Gesundheitsökonomie, Charité - Universitätsmedizin Berlin, Berlin, Deutschland
}

Die Integrative Medizin (IM) hat sich, begrifflich ursprünglich aus den USA kommend, mittlerweile auch in Europa über die letzten 20 Jahre fest etabliert. Dabei handelt es sich um ein relativ neues medizinisches Fachgebiet und begleitendes Paradigma, das sich an vorbestehenden Bereichen tradierter Medizinsysteme - vor allem aus dem Bereich der Naturheilkunde und Komplementärmedizin - anlehnt, diese jedoch mit etablierten Verfahren der konventionellen Medizin kombiniert und über ganzheitliche, evidenzinformierte und patientenzentrierte Elemente verfügt. Allerdings erfährt die bisherige Beschreibung der IM aktuell ein weiteres "Update".

In den USA hat die IM zusammen mit der "Mind-Body-Medizin" erst dem Bereich der Complementary and Alternative Medicine (CAM) eine anerkannte Sichtbarkeit und Verankerung auch an den großen und etablierten akademisch-medizinischen Einrichtungen, wie z.B. der Harvard Medical School oder der Stanford University School of Medicine, gegeben, so dass heute nach Etablierung des neuen Begriffs der IM zumeist von Complementary and Integrative Medicine (CIM) gesprochen wird.

Die wissenschaftlich-akademische IM ist in Nordamerika u.a. im sogenannten Academic Consortium organisiert (www.imconsortium.org). An der US-amerikanischen Gesundheitsadministration (National Institutes of Health - eine zentrale Behörde des US-Gesundheitsministeriums) gibt es eine gut sichtbare Abteilung namens "National Center for Complementary and Integra- tive Health (NCCIM)", die aus dem "National Center for Complementary \& Alternative Medicine (NCCAM)" hervorgegangen ist. Institute, Lehrstühle, Aus- und Weiterbildungseinrichtungen sowie Kliniken und Ambulanzen zur Anwendung der IM sind in Nordamerika an vielen Standorten entstanden: Dank einer intensiven staatlichen Forschungsförderung des NCCAM und der damit verknüpften regen Forschungsleistung wächst das Wissen zur komplementären und IM kontinuierlich an.

In Mitteleuropa und insbesondere im deutschsprachigen Raum gibt es eine lange Tradition der Naturheilkunde. Die Konzepte der modernen Naturheilkunde gehen auf die antike Medizin und die wissenschaftlichen Bestrebungen in Theorie-, Grundlagen- und Anwendungsforschung zurück. Allerdings hat der Bereich der Naturheilkunde und der Komplementärmedizin in Mitteleuropa kein vergleichbares Wachstum wie in Nordamerika in den letzten knapp 30 Jahren erlebt, auch weil hier die staatliche Forschungsförderung nicht ausgeprägt ist. Die verschiedenen Therapie- sowie Theorierichtungen aus dem Bereich der Naturheilkunde bzw. der Komplementärmedizin tun sich schwer, eine gemeinsame Basis mit der IM oder Mind-Body-Medizin zu finden.

Dennoch sind auch bei uns in Europa in den letzten 10 Jahren mehrere Lehrstühle, Professuren und akademische Institute sowie klinische stationäre und ambulante Einrichtungen entstanden, die entweder den Begriff "Naturheilkunde" oder "Komplementäre Medizin", "Integrative Medizin" bzw. "Integrative Gesundheit", "Inte- 
grative Gesundheitsförderung” oder "Integrative Gesundheitsversorgung" im Namen tragen. Ganz eindeutig: Die IM ist nicht nur bei den Patienten und Ärzten, sondern auch in der akademischen Welt angekommen. Sie wird verstärkt auch von allen Beteiligten des Gesundheitswesens wahrgenommen, und mittlerweile gibt es immer mehr universitäre Kooperationen. In diesem Kontext sei exemplarisch auf das Ergebnis der Schweizer Verfassungsinitiative von 2009 hingewiesen: Bevölkerung und Standesvertretungen haben damals den neuen Verfassungsartikel zur Komplementärmedizin angenommen - dieser verpflichtet Bund und Kantone, im Rahmen ihrer Zuständigkeiten dafür zu sorgen, dass Verfahren der Komplementärmedizin in das Schweizer Gesundheitssystem integriert werden. Auch sei als Meilenstein der 1. World Congress Integrative Medicine and Health (WCIMH) 2017 in Berlin genannt, in dessen Rahmen das sogenannte Berlin Agreement [1] mit dem Titel "Self-Responsibility and Social Action in Practicing and Fostering Integrative Medicine and Health Globally" formuliert wurde, wo es um den Versuch eines einheitlichen Verständnisses für Ärzte und weitere Gesundheitsberufe dieses Fachgebietes geht. Im Jahr 2021 wird der 2. WCIMH in Florenz, Italien, stattfinden, bei dem das Berlin Agreement gegebenenfalls eine revidierte Neuauflage erhält.

Aber es gibt auch Widerstände gegen diese Entwicklung: Die aktuellen Diskussionen beispielsweise rund um das sogenannte "Münsteraner Memorandum" [2] sowie um Abschaffungen von Zusatzbezeichnungen aus dem Bereich der Komplementärmedizin wie auch die nach wie vor geringe Zurverfügungstellung von Forschungsmitteln zur wissenschaftlichen Untersuchung und Etablierung von Integrativer- und Komplementärmedizin seien in diesem Kontext genannt. Und nicht zuletzt sei auch auf die leidenschaftlich geführten fachinternen Diskussionen zu Definitionen, Begriffen, Zielgruppen, Platzierungen im Gesundheitswesen und Indikationen einer IM verwiesen - sowie auf die noch genauer auszuhandelnden Beziehungen der verschiedenen beteiligten Disziplinen und "Health Professionals" untereinander. Diese Entwicklungen weisen darauf hin, wie wichtig und zeitgemäß es ist, die Diskussionen um eine IM der Zukunft auf eine größere, sichtbare und für alle Interessierten zugängliche Bühne zu heben.

Allein die Frage, ob und warum es den Begriff - oder das Fachgebiet - der IM gibt und wie sie definiert werden kann, wird kontrovers diskutiert. Wir hören heute von einer zunehmenden Zahl von Subspezialisierungen im Gebiet, wie der "Integrativen Onkologie", "Integrativen Schmerztherapie", "Integrativen Pädiatrie", "Integrativen Psychiatrie", "Integrativen Kardiologie" oder "Integrativen Allgemeinmedizin", aber eine eindeutige Definition dieses Begriffs ist nach wie vor eher "work in progress", obwohl von essenzieller Bedeutung für die Weiterentwick- lung des gesamten Bereiches, insbesondere, aber nicht nur, im akademischen Kontext. Dabei sind Fragen der Abrechnung und Vergütung im Gesundheitssystem, der konkreten Versorgungsstrukturen bzw. -leistungen sowie der Legitimation der verschiedenen Gesundheitsberufe, die an der erfolgreichen Umsetzung der IM oder Mind-BodyMedizin beteiligt sind, ebenfalls zu berücksichtigen.

Zentral - und allen Strömungen der IM gemein - ist, dass, neben dem traditionellen Bezug zu Komplementärmedizin und Naturheilkunde, die Rolle des aktiven und zur Selbstfürsorge befähigten Patienten gestärkt und auf seine persönlichen Gesundheits- und Heilungskompetenzen fokussiert werden soll. Im Sinne eines ganzheitlichen und ressourcenorientierten Ansatzes wird, neben einer pathogenetischen Sichtweise, die salutogene und gesundheitsförderliche Perspektive ergänzt bzw. in das bestehende Paradigma integriert. Eine Patientenzentrierung, wie sie heute auch für die konventionelle Medizin gefordert wird [3], ist in der "Matrix" der IM von vornherein angelegt, zusätzlich zum pluralistischen und multiprofessionellen, oftmals teambasierten, Versorgungsmodell. Auch durch diese Entwicklungen ist die IM nun mehr als anschlussfähig geworden zu innovativen Konzepten einer modernen und umfassenden Gesundheitsversorgung oder "Heilkunst von morgen", auf jeden Fall aber ist sie heute weit mehr als ein reines Addieren, d.h. Zusammenbringen, von "Komplementärmedizin und Schulmedizin". Auch der Digitalisierung und sinnvollen Anwendung von Informationstechnologien verschließt sie sich nicht, im Gegenteil: Die IM ist im "Innovation Lab" einer hochmodernen Medizin angekommen und vereint in besonderer Weise das Heilwissen von der Antike bis heute mit einer ganzheitlichen Heilkunst der $\mathrm{Zu}$ kunft, die sich auch modernsten Techniken sowie den Grundsätzen einer demokratisch-ökologischen Werteordnung nicht verschließt. Dieses spiegelt sich auch in ihrer jüngsten Definition wider [4], die wir hier erstmalig in diesem Journal vorstellen:

"Integrative Medicine and Health' reaffirms the importance of the relationship between practitioner and patient, focuses on the whole person, is informed by evidence, and makes use of all appropriate therapeutic, preventive, health-promoting, or lifestyle approaches, healthcare professionals and disciplines, to achieve optimal health and healing, emphasizing the art and science of healing. It is based on a social and democratic as well as natural and healthy environment."

“'Integrative Medizin und Gesundheit' bekräftigt die Bedeutung der Beziehung zwischen Arzt und Patient, zielt auf die ganze Person ab, wird durch Evidenz informiert und bedient sich aller geeigneten therapeutischen, präventiven, gesundheitsfördernden oder Lifestyle-Ansätze, Fachkräfte und Disziplinen des Gesundheitswesens, um eine optimale Gesundheit und Heilung zu erreichen - Kunst und Wissenschaft des Heilens gleichermaßen hervorhebend. Sie basiert auf einer sozialen und demokratischen sowie natürlichen und gesunden Umwelt."
Esch/Brinkhaus 


\section{Literatur}

Neue Definitionen der Integrativen Medizin
1 Organizing Committee, World Congress on Integrative Medicine and Health. The Berlin Agreement: Self-Responsibility and Social Action in Practicing and Fostering Integrative Medicine and Health Globally. J Altern Complement Med. 2017;23(5):320-1.

2 Münsteraner Kreis. Münsteraner Memorandum Heilpraktiker [Internet]. [cited 2017 Aug 21]. Available from: http://daebl.de/ BB36
3 Esch T. OpenNotes, Patient Narratives, and Their Transformative Effects on Patient-Centered Care. NEJM Catalyst. 2018 Oct 4.

4 Brinkhaus B, Esch T. Integrative Medizin und Gesundheit - Konstrukt einer modernen Medizin. In: Brinkhaus B, Esch T, editors. Integrative Medizin und Gesundheit. Berlin: Medizinisch Wissenschaftliche Verlagsgesellschaft Berlin; 2020, in press. 